\title{
Nucleus accumbens activation mediates the influence of reward cues on financial risk taking
}

\author{
Brian Knutson ${ }^{\mathrm{a}}$, G. Elliott Wimmer ${ }^{\mathrm{a}}$, Camelia M. Kuhnen ${ }^{\mathrm{c}}$ and Piotr Winkielman ${ }^{\mathrm{b}}$ \\ ${ }^{a}$ Department of Psychology, Stanford University, Stanford, 'D Department of Psychology, University of California, San Diego, California and \\ 'Kellogg School of Management, Northwestern University, Evanston, Illinois, USA \\ Correspondence to Dr Brian Knutson, PhD, Department of Psychology, Stanford University, Building 420, Jordan Hall, Stanford, \\ CA 94305, USA \\ Tel: + I 650724 2965; fax: + I 650725 5699; e-mail: knutson@psych.stanford.edu
}

Received 9 January 2008; accepted 15 January 2008

In functional magnetic resonance imaging research, nucleus accumbens (NAcc) activation spontaneously increases before financial risk taking. As anticipation of diverse rewards can increase NAcc activation, even incidental reward cues may influence financial risk taking. Using event-related functional magnetic resonance imaging, we predicted and found that anticipation of viewing rewarding stimuli (erotic pictures for 15 heterosexual men) increased financial risk taking, and that this effect was partially mediated by increases in NAcc activation. These results are consistent with the notion that incidental reward cues influence financial risk taking by altering anticipatory affect, and so identify a neuropsychological mechanism that may underlie effective emotional appeals in financial, marketing, and political domains. NeuroReport 19:509-513 (c) 2008 Wolters Kluwer Health | Lippincott Williams \& Wilkins.

Keywords: accumbens, cue, decision, financial, functional magnetic resonance imaging, human, reward, risk, striatum

\section{Introduction}

Recent research suggests that affect changes during anticipation of, as well as in response to, goal outcomes [1-3]. Functionally, 'anticipatory affect' might promote goaldirected behavior. Anticipatory affect, however, might also subvert goal-directed behavior when elicited by incidental stimuli. Here, we examined whether incidentally elicited anticipatory affect influences financial risk taking, and characterized neuropsychological correlates of this influence.

Event-related functional magnetic resonance imaging (fMRI) research has implicated activation of the nucleus accumbens (NAcc) as a neural marker of positive arousal (PA; feelings like 'excitement'), as anticipation of both financial [2,4] and nonmonetary rewards (e.g. erotic pictures) $[5,6]$ increases NAcc activation. Conversely, activation of the insula has been implicated as a neural marker of negative arousal (NA; feelings like 'anxiety'), as anticipation of both financial [7] and nonmonetary punishments (e.g. pictures of snakes and spiders) [8] increases insular activation. Currently, however, it is not clear whether insular activation specifically marks NA or general arousal [9].

Anticipatory affect might influence financial risk taking by modifying the salience of potential gains or losses. In finance, risk (or variance in outcomes) increases proportional to the magnitude of anticipated gains and losses [10]. All other inputs being equal (e.g. information and incentives), PA should increase the salience of potential gains, and thus increase subsequent risk taking, whereas NA should increase the salience of potential losses, and thus decrease subsequent risk taking. Indeed, in an investment task, endogenous NAcc activation predicted shifts to high- risk options, whereas insular activation predicted shifts to low-risk options [7]. Anticipatory affect should influence risk taking independent of its source, and particularly when circumstances are uncertain or strategies are changing (i.e. people decide to change rather than repeat a past choice) [7].

This study explored the influence of positive stimuli on financial risk taking by examining whether: (i) incidental positive stimuli would increase shifts to a high-risk option; (ii) NAcc activation would increase before shifts to a highrisk option; and (iii) NAcc activation would mediate the influence of incidental positive stimuli on subsequent highrisk shifts.

\section{Methods}

Participants

Fifteen healthy right-handed (self-reported) heterosexual men (age mean=20.73; $\mathrm{SD}=2.12$; range, 18-26) participated. Along with typical magnetic resonance exclusions (e.g. metal in the body), participants were screened for psychotropic drugs and ibuprofen, substance abuse in the past month, and history of psychiatric disorders (Diagnostic and Statistical Manual Of Mental Disorder-IV Axis I) and gave informed consent. Participants received $\$ 20.00$ per hour for participating as well as $\$ 10.00$ cash endowment plus their earnings (positive or negative) from gambling during the task.

Task

Participants played a practice version of the task before entering the scanner, during which they learned the associations between shapes and pictures (on which they 


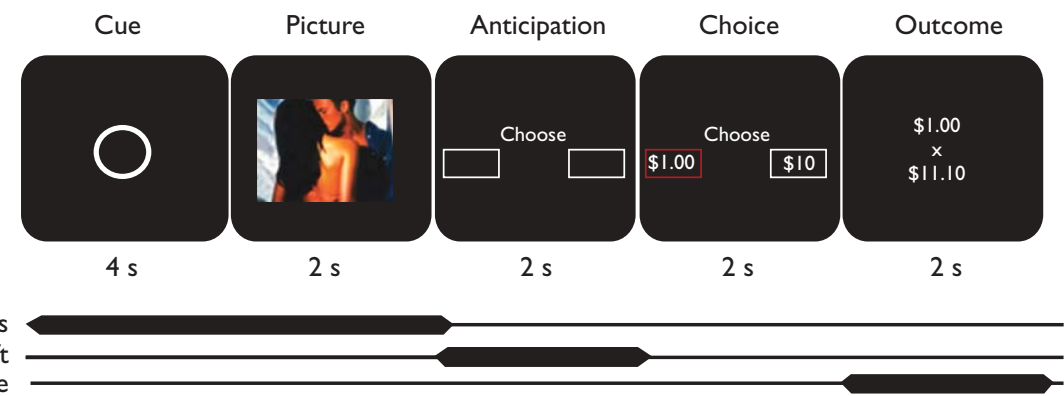

Fig. I Cued risk task structure and regressor timing. Participants first viewed affective stimuli consisting of a shape (cue: circle, triangle, square) followed by a picture (picture: erotic couples, household appliances, snakes and spiders). Next, participants gambled by first waiting (anticipation), next choosing the high or low-risk option (choice), and finally viewing the outcome of their choice (outcome). Conjoined regressors modeled brain activation in response to affective stimuli (cue + picture) and during anticipation of choosing the gamble (anticipation).

were explicitly tested), and were instructed that these stimuli were unrelated to the outcomes of subsequent gambles. To cleanly isolate within-subject shifts in financial risk taking, gambles featured equal expected value (i.e. $\$ 0.00$, as each involved potential gains or losses) but different outcome variances (i.e. $50 \%$ probability of gaining or losing either $\$ 1.00$ or $\$ 0.10$; Fig. 1$)$.

During functional scanning, the task included 54 trials in total (i.e. 18 positive, neutral, and negative). During the first part of each trial, participants saw visual stimuli. These consisted of one of three shape cues (i.e. circle, square, or triangle; $4 \mathrm{~s}$ ) signaling the impending display of a positive (i.e. erotic couples), negative (i.e. snakes or spiders), or neutral (i.e. household appliances) picture, respectively ( $2 \mathrm{~s})$. The cue/picture stimulus combination was designed to maximize anticipatory affect, and participants were asked to indicate the appearance of each picture with a button press. During the second part of each trial participants gambled. First, they waited while viewing two empty boxes ( $2 \mathrm{~s})$, then chose either a high (1.00) or low (0.10) risk financial gamble ( $2 \mathrm{~s}$, randomly appearing in left vs. right boxes), and finally saw the outcome of their choice for that trial as well as their cumulative earnings ( $2 \mathrm{~s})$. After scanning, participants rated their reactions to each picture on dimensions of valence and arousal (subsequently mean-deviated within subject and rotated $45^{\circ}$ to derive independent ratings of PA and NA for each picture, as described in [11]). Reaction time to picture appearance and the choice prompt was log-transformed before analysis.

\section{Functional magnetic resonance imaging acquisition} and analysis

Images were acquired with a 1.5-T General Electric MRI scanner (General Electric, Milwaukee, Wisconsin, USA) and a standard quadrature head coil. Twenty-four contiguous axial 4-mm-thick slices (in-plane resolution $3.75 \times 3.75 \mathrm{~mm}$ ) extended axially from the mid-pons to the top of the skull. Functional scans were acquired with a T2*-sensitive spiral in/out pulse sequence (repetition time $=2 \mathrm{~s}$, echo time $=$ $40 \mathrm{~ms}$, flip $=90^{\circ}$ ) [12]. High-resolution structural scans for localization and coregistration of functional data were acquired with a T1-weighted spoiled grass sequence (repetition time $=100 \mathrm{~ms}$, echo time $=7 \mathrm{~ms}$, flip $=90^{\circ}$ ). Analyses utilized AFNI software (National Institute of Health, Bethesda, Maryland, USA) [13]. For preprocessing, data were sinc interpolated, concatenated across runs, motion- corrected, spatially smoothed (Full-Width Half-Maximum $=4 \mathrm{~mm})$, high-pass filtered $(>0.01 \mathrm{~Hz})$, and normalized to percent signal change relative to the task voxel mean.

Localization analyses utilized multiple regression in which regressors of interest contrasted: (i) positive versus negative stimuli (i.e. cue + picture combined, which controls for arousal); (ii) anticipation of choosing the high versus low-risk option (anticipation); (iii) anticipation of shifting to the high versus low-risk option (anticipation); and (iv) highrisk gain versus loss outcomes (outcome; Fig. 1). These were orthogonalized and convolved with a $\gamma$-variate model of the hemodynamic response function before entry in the model [14]. Regressors of noninterest indexed choice reaction time, residual motion (six parameters), and baseline, linear, and quadratic trends. Regressor of interest coefficient maps were coregistered with structural maps, spatially normalized, and submitted to a one-sample $t$-test to test for random effects (a priori NAcc volumes of interest (VOIs) $P<0.01$ uncorrected; cluster $=34 \mathrm{~mm}^{3}$ voxels).

Prediction analyses utilized VOI percent signal change time course peaks (from $8 \mathrm{~mm}$ diameter spherical VOIs identified in earlier studies) to predict choice and shifts $[7,15,16]$. Logistic regressions analyzed whether NAcc (Talairach coordinates: $\pm 12,10,-2$ ) and right insula (Talairach coordinates: $39,20,10$ ) activation during choice anticipation (lagged by $4 \mathrm{~s}$ ) predicted subsequent decisions to choose or shift to the high-risk option, both before and after controlling for experimental (e.g. positive stimuli) and control variables (i.e. cumulative earnings, preceding outcome).

Mediation analyses utilized VOI data from prediction analyses [17]. For the independent variable, positive stimuli were assigned a weight of 1 , negative stimuli a weight of -1 , and neutral stimuli a weight of 0 . For the dependent variable, high-risk shifts were assigned a weight of 1 and low-risk shifts were assigned a weight of -1 . Covariates included effects of cumulative earnings, winning on the previous trial, and right insula activation. The mediator was peak NAcc activation during choice anticipation (4s lag). To verify mediation, path significance was assessed using directional hypotheses $(P<0.05$, one-tailed).

\section{Results}

Behavioral analyses indicated that positive stimuli increased self-reported positive arousal $(1.97 \pm 0.205)$ and 
negative stimuli increased self-reported negative arousal $(1.32 \pm 0.153)$, relative to neutral stimulus-induced positive arousal $(-1.42 \pm 0.138)$ and negative arousal $(-1.39 \pm 0.139$, $P<0.001)$. Positive stimuli also increased subsequent highrisk choices and shifts to the high-risk option $(67.8 \pm 3.84$ and $61.9 \pm 3.19 \%)$, but negative stimuli did not $(59.3 \pm 3.97$ and $42.7 \pm 4.38 \%)$, relative to neutral stimuli $(57.40 \pm 4.26$ and $39.1 \pm 4.77 \% ; P<0.01)$. Log-transformed mean reaction time to pictures did not differ as a function of stimulus type (i.e. positive, negative, and neutral). Log-transformed mean reaction time to the choice prompt did not differ as a function of earlier stimulus type (as above) or choice type (i.e. high vs. low risk, shift vs. stay).

Localization analyses using multiple regression indicated that brain activation correlated with viewing positive versus negative stimuli in a number of regions including mesial prefrontal cortical and ventral striatal subcortical regions (e.g. NAcc, putamen) and posterior cingulate regions, as predicted. Anticipation of shifting to the high-risk option versus shifting to the low-risk option correlated with activation in the bilateral NAcc and caudate as well as deactivation of the right anterior insula, as predicted [7]. Conjunction of these contrasts yielded only NAcc activation (Fig. 2). Replicating previous findings [15], gain versus loss high-risk outcomes correlated with activation in the mesial prefrontal cortical, caudate, putamen, and posterior cingulate (Table 1).

Prediction analyses utilized logistic regressions to determine whether brain activation could predict financial risk taking. The first analysis indicated that viewing positive stimuli predicted subsequent shifts to the high-risk option, but gains on earlier high-risk trials predicted shifts to the low-risk option. A second analysis indicated that bilateral NAcc activation significantly predicted subsequent shifts to the high-risk option. A third analysis including stimulus and brain activation variables together indicated that viewing positive stimuli no longer significantly predicted shifts to the high-risk option, but NAcc activation did, suggesting a critical role for NAcc activation (Table 2). The Akaike Information Criterion indicated that the increased fit of this model was not solely owing to increased parameters. Analyses including all choices (rather than just shifts) revealed a similar but less robust pattern of results, as predicted.

Mediation analyses evaluated the directional prediction that NAcc activation might mediate the influence of positive stimuli on shifts to the high-risk option. Bootstrapped mediation revealed significant paths from positive stimuli to NAcc activation and from NAcc activation to shifts to the high-risk option. The direct path from positive stimuli to shifts to the high-risk option was also significant, but less so after controlling for indirect paths incorporating NAcC activation (Fig. 3). The NAcc was the only region examined whose activation both predicted shifting to the high-risk option and also mediated the influence of positive stimuli on high-risk shifts.

Previous analyses controlled for individual differences by incorporating fixed effects into models. An anticipatory affect account, however, further predicts that individuals who experience greater self-reported positive arousal in response to positive stimuli should make more shifts to the high-risk option. The correlation between individual mean

Table I Brain activation correlated with exposure to positive versus negative stimuli (i.e. cue + picture), during anticipation of switching to a high versus low-risk option, and in response to gain versus loss high-risk outcomes

\begin{tabular}{|c|c|c|c|c|}
\hline & Peak Z & $R$ & $A$ & $S$ \\
\hline \multicolumn{5}{|l|}{ Positive $>$ negative stimuli } \\
\hline L subgenual cingulate & 4.09 & -8 & 38 & - II \\
\hline R subgenual cingulate & 3.86 & II & 23 & - II \\
\hline LOFC & 3.91 & -26 & 19 & - II \\
\hline L caudate head & 3.97 & -4 & 15 & I \\
\hline L NAcc* & 3.09 & -12 & II & -4 \\
\hline R NAcc* & 3.22 & 7 & 8 & -6 \\
\hline R putamen & 3.89 & 19 & 8 & -6 \\
\hline R middle frontal gyrus, BA 6 & 4.10 & 33 & 0 & 42 \\
\hline R middle frontal gyrus, BA 9 & 4.34 & 49 & 4 & 38 \\
\hline L middle temporal gyrus & 3.96 & -56 & -56 & 8 \\
\hline $\mathrm{L}$ middle temporal gyrus & 4.42 & -49 & -53 & 4 \\
\hline L posterior cingulate & 3.89 & -4 & -56 & 16 \\
\hline $\mathrm{R}$ middle temporal gyrus & 4.09 & 38 & -60 & 19 \\
\hline L middle occipital gyrus & 3.94 & -42 & -83 & 16 \\
\hline \multicolumn{5}{|l|}{ High > low-risk shift } \\
\hline $\mathrm{R}$ anterior insula* & -2.38 & 33 & 22 & II \\
\hline L caudate* & 3.44 & -8 & 22 & 0 \\
\hline R caudate* & 3.79 & 4 & 12 & 4 \\
\hline L NAcc* & 3.29 & -12 & 4 & -6 \\
\hline R NAcc* & 3.45 & 12 & 6 & -6 \\
\hline \multicolumn{5}{|l|}{ Gain versus loss outcome } \\
\hline MPFC* & 3.34 & 0 & 62 & 5 \\
\hline L caudate* & 3.52 & -15 & 12 & 8 \\
\hline R putamen* & 3.65 & 18 & 8 & -3 \\
\hline L putamen* & 3.38 & -18 & 8 & -3 \\
\hline $\mathrm{R}$ inferior frontal gyrus & 3.73 & 45 & 8 & 27 \\
\hline L precentral gyrus & 3.73 & -56 & 0 & 4 \\
\hline Posterior cingulate* & 3.55 & 0 & -53 & 15 \\
\hline R lingual gyrus & 3.74 & 22 & -75 & -6 \\
\hline
\end{tabular}

L, left; MPFC, mesial prefrontal cortical; NAcc, nucleus accumbens; OFC, orbitofrontal cortex; $\mathrm{R}$, right.

* Predicted region significant at $P<0.005$ corrected, cluster $>$ two $4 \mathrm{~mm}^{3}$ voxels; other regions significant at $P<0.00$ l uncorrected, cluster $>$ two $4 \mathrm{~mm}^{3}$ voxels.

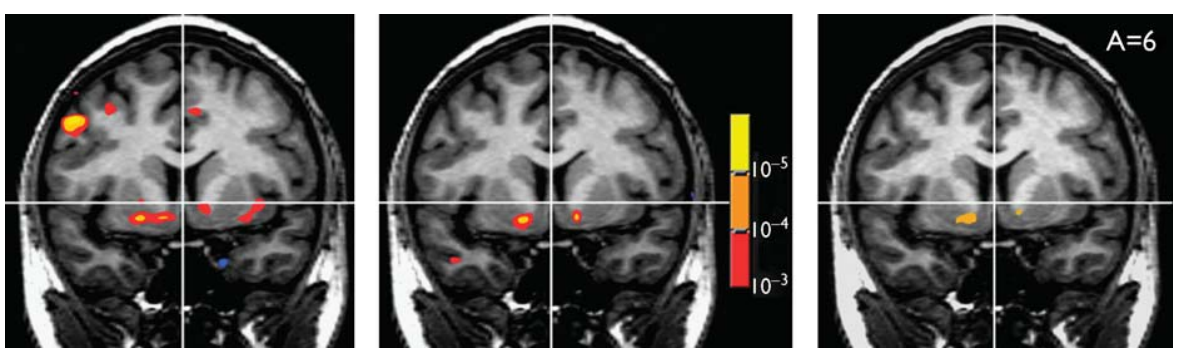

Fig. 2 Brain activation associated with viewing positive versus negative stimuli (left), with anticipation of shifting to the high-risk option versus shifting to the low-risk option (middle), and with their conjunction (right; $P<0.01$, two-tailed, uncorrected). 
Table 2 Logistic regressions predicting shifts in the cued risk task $(n=15)$

\begin{tabular}{lccc}
\hline & Stimulus & Brain & Combined \\
\hline Constant & 0.97 & 1.47 & 1.42 \\
& $0.98(1.010)$ & $1.50(1.02)$ & $1.46(1.029)$ \\
Cumulative earnings & 0.11 & 0.25 & 0.05 \\
& $0.01(0.075)$ & $0.02(0.075)$ & $0.00(0.075)$ \\
Preceding outcome & $-7.40^{* * *}$ & $-7.75 * * *$ & $-7.54^{* * * *}$ \\
& $-2.01(0.271)$ & $-2.19(0.283)$ & $-2.14(0.284)$ \\
Positive stimulus & $2.00^{*}$ & & 1.75 \\
NAcc (bilateral) & $0.57(0.283)$ & & $0.50(0.286)$ \\
& & $2.74^{* * *}$ & $2.59 * *$ \\
Insula (right) & -1.15 & $1.14(0.416)$ & $1.09(0.419)$ \\
& $-0.44(0.378)$ & $-0.39(0.378)$ & \\
Number of observations & 315 & 315 & 315 \\
Pseudo- $R^{2}$ & 0.169 & 0.178 & 0.186 \\
AIC & 398.6 & 396.3 & 395.3 \\
\hline AIC Akaik & &
\end{tabular}

AIC, Akaike Information Criterion; NAcc, nucleus accumbens. Regressions included subject fixed effects. No participants were, however, significant at $P<0.01$ and omission of fixed effects did not affect the results. $Z$-scores above coefficients with standard errors in parentheses. Significance: $*<0.05 ; * *<0.01 ; * *<0.001$, two-tailed.

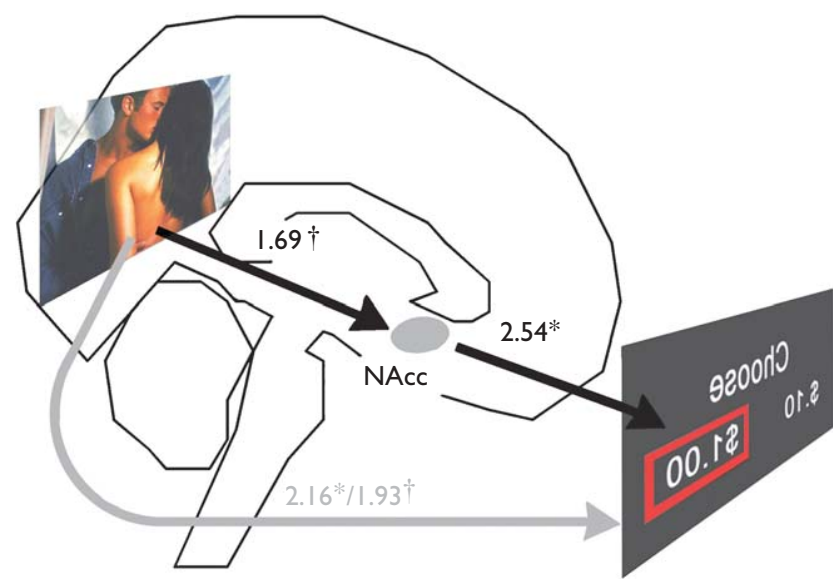

Fig. 3 Anticipatory nucleus accumbens (NAcc) activation partially mediates the influence of positive stimuli on subsequent shifts to the highrisk option ( $t$-scores above paths, $* P<0.025, \dagger P<0.05$; one-tailed). Bootstrapped (robust; $n=1000$ ) mediation analysis indicated a significant path from positive stimuli to NAcc activation $[\beta=0.037, \operatorname{SEM}=0.022$; $t(315)=1.69, P<0.05$, one-tailed] and a significant path from NAcc activation to high-risk shifts $[\beta=0.41$, SEM $=0.162 ; t(315)=2.54, P<0.05$, onetailed]. The path from positive stimuli to high-risk shifts was also significant $[\beta=0.137, \mathrm{SEM}=0.063 ; t(315)=2.16, P<0.05$, one-tailed], but less so $[\beta=0.121, \mathrm{SEM}=0.063 ; t(315)=1.93, P<0.05$, one-tailed $]$ after adding indirect paths involving NAcc activation to the model. Bias corrected and accelerated confidence intervals (Cls) verified the significance of this partial mediation $(\mathrm{Cl}$ bounds $=0.0002-0.0447)$. Of the model covariates (i.e. cumulative earnings, anterior insula activation), only losses on the previous trial $[t(315)=-9.14, P<0.001]$ significantly predicted shifts to the high-risk option.

self-reported positive arousal to the positive stimuli and proportion of high risk to total shifts was significant $(r=0.70$, $P<0.01)$, whereas the correlation of mean self-reported negative arousal to the positive stimuli and proportion of low risk to total shifts was not significant.

\section{Discussion}

This study investigated whether incidental reward cues can influence financial risk taking and sought to identify underlying neural mechanisms. Positive stimuli increased shifting to a high-risk option, and this behavioral influence was partially mediated by NAcc activation. Further, individual differences in self-reported positive arousal in response to positive stimuli predicted the strength of these effects. Together, these results suggest that even incidental reward cues can act on anticipatory affect to alter financial risk taking. The findings have broad implications for understanding how affect might influence decisions, and for assessing the effectiveness of emotional persuasive techniques.

The findings provide an initial demonstration that incidental external stimuli can influence subsequent financial risk taking, and that brain activation in a specific region mediates this behavioral effect. Other studies have correlated spontaneous (or endogenous) activation in related brain regions with subsequent decisions. These experiments have focused on investing [7], learning [18], or gambling $[19,20]$ tasks, in which earlier feedback could potentially provide domain-specific information about the next best choice. In this study, however, affective stimuli had no explicit or implicit relationship to subsequent gambles, and so could not inform the next choice. Still, positive stimuli influenced subsequent choice, and did so partially as a function of NAcc activation. Combined with earlier demonstrations that NAcc activation correlates with stimuluselicited positive arousal, this evidence is consistent with the notion that anticipatory affect has the capacity not only to facilitate, but also to subvert decisions.

Importantly, a conjunction analysis indicated that only the NAcc was activated both by positive stimuli and by anticipation of shifting to the high-risk option. NAcc activation does not increase solely in response to reward cues, as spontaneous changes in NAcc activation predicted risky shifts in an earlier study [7]. The findings also could not be accounted for in terms of motor preparation, because peak activation was modeled during anticipation, when participants saw two boxes and knew that the high-risk option would appear in one and the low-risk option in the other, but did not know which option would appear in which box (also, reaction time did not differ between high and low-risk choices or shifts). A 'switching' account predicts that NAcc activation should increase before shifting from a repeated choice to any new choice [21], but not that NAcc activation should preferentially increase before shifts to the high-risk but not the low-risk option.

\section{Conclusion}

Incidental reward cues can influence financial risk taking, and may do so in part by activating the NAcc. From a financial standpoint, these results imply that anticipatory affect may alter the perception of rewards, and the tendency to weigh them against risks $[7,22]$. Thus, these findings may lead to methods of determining when persuasive appeals should and should not work - whether they appeal to passion or to reason.

\section{Acknowledgements}

The authors thank Larry Ozowara, George Wang, and Cliff Baum for assistance in stimulus construction, experimental design, and data acquisition. They also thank Antonio Rangel and Adam Aron for feedback on previous drafts. 
This research was supported by NIH grant DA020615-01 to B.K.

\section{References}

1. Loewenstein GF, Weber EU, Hsee CK, Welch N. Risk as feelings. Psychol Bull 2001; 2:267-286

2. Knutson B, Adams CM, Fong GW, Hommer D. Anticipation of increasing monetary reward selectively recruits nucleus accumbens. J Neurosci 2001; 21:RC159.

3. Bechara A, Tranel D, Damasio H, Damasio AR. Failure to respond autonomically to anticipated future outcomes following damage to prefrontal cortex. Cereb Cortex 1996; 6:215-225.

4. Preuschoff K, Bossaerts P, Quartz SR. Neural differentiation of expected reward and risk in human subcortical structures. Neuron 2006; 51: 381-390.

5. Hamann S, Herman RA, Nolan CL, Wallen K. Men and women differ in amygdala response to visual sexual stimuli. Nat Neurosci 2004; 7:411-416.

6. Sabatinelli D, Bradley MM, Lang PJ, Costa VD, Versace R. Pleasure rather than salience activates human nucleus accumbens. J Neurophysiol 2007; 98:1374-1379.

7. Kuhnen CM, Knutson B. The neural basis of financial risk-taking. Neuron 2005; 47:763-770.

8. Nitschke JB, Sarinopoulos I, Mackiewicz KL, Schaefer HS, Davidson RJ. Functional anatomy of aversion and its anticipation. NeuroImage 2006; 29:106-116.

9. Wager TD, Phan KL, Liberzon I, Taylor SF. Valence, gender, and lateralization of functional brain anatomy in emotion: a meta-analysis of findings from neuroimaging. NeuroImage 2003; 19:513-531.
10. Markowitz H. Portfolio selection. J Finance 1952; 7:77-91.

11. Knutson B, Taylor J, Kaufman M, Peterson R, Glover G. Distributed neural representation of expected value. J Neurosci 2005; 25:4806-4812.

12. Glover GH, Law CS. Spiral-in/out BOLD fMRI for increased SNR and reduced susceptibility artifacts. Magn Reson Med 2001; 46:515-522.

13. Cox RW. AFNI: software for analysis and visualization of functional magnetic resonance images. Comput Biomed Res 1996; 29:162-173.

14. Zarahn E. Testing for neural responses during temporal components of trials with BOLD fMRI. NeuroImage 2000; 11:783-796.

15. Knutson B, Fong GW, Bennett SM, Adams CM, Hommer D. A region of mesial prefrontal cortex tracks monetarily rewarding outcomes: characterization with rapid event-related FMRI. NeuroImage 2003; 18:263-272.

16. Knutson B, Rick S, Wimmer GE, Prelec D, Loewenstein G. Neural predictors of purchases. Neuron 2007; 53:147-156.

17. Preacher KJ, Hayes AF. SPSS and SAS procedures for estimating indirect effects in simple mediation models. Behav Res Methods Instrum Comput 2004; 36:717-731.

18. Hampton AN, O'Doherty JP. Decoding the neural substrates of rewardrelated decision making with functional MRI. Proc Natl Acad Sci 2007; 104:1377-1382.

19. Cohen MX, Ranganath C. Behavioral and neural predictors of upcoming decisions. Cogn Affect Behav Neurosci 2005; 5:117-126.

20. Matthews SC, Simmons AN, Lane SD, Paulus MP. Selective activation of the nucleus accumbens during risk-taking decision making. NeuroReport 2004; 15:2123-2127.

21. Robbins TW, Everitt BJ. Neurobehavioral mechanisms of reward and motivation. Curr Opin Neurobiol 1996; 6:228-236.

22. Knutson B, Bossaerts P. Neural antecedents of financial decisions. J Neurosci 2007; 27:8174-8177. 